\title{
Effects of internal target structures on laser-driven neutron production
}

\author{
Yihang Zhang, Wei-Min Wang, Yutong Li, Zhe Zhang, \\ Paul McKenna, David Neely and Jie Zhang
}

\section{Published version information}

Citation: Y Zhang et al. "Effects of internal target structures on laser-driven neutron production." Nuclear Fusion, vo. 59, no. 7 (2019): 076032.

DOI: $\underline{10.1088 / 1741-4326 / a b 1 c d a}$

This is the Accepted Manuscript version of an article accepted for publication in Nuclear Fusion. IOP Publishing Ltd is not responsible for any errors or omissions in this version of the manuscript or any version derived from it. The Version of Record is available online at DOI above.

This version is made available in accordance with publisher policies. Please cite only the published version using the reference above. This is the citation assigned by the publisher at the time of issuing the AAM. Please check the publisher's website for any updates. 


\title{
Effects of internal target structures on laser-driven neutron production
}

\author{
Yihang Zhang ${ }^{1,3}$, Win-Min Wang ${ }^{1,4,6 *}$, Yutong $\mathbf{L i}^{1,3,7 *}$, Zhe \\ Zhang $^{1}$, Paul McKenna ${ }^{2}$, David Neely ${ }^{2,5}$ and Jie Zhang ${ }^{1,4,8}$ \\ ${ }^{1}$ Beijing National Laboratory for Condensed Matter Physics, Institute of Physics, \\ Chinese Academy of Sciences, Beijing 100190, China \\ ${ }^{2}$ Department of Physics, Scottish Universities Physics Alliance (SUPA), University \\ of Strathclyde, Glasgow, G4 0NG, UK \\ ${ }^{3}$ School of Physical Sciences, University of Chinese Academy of Sciences, Beijing \\ 100049, China \\ ${ }^{4}$ Collaborative Innovation Center of IFSA (CICIFSA), Shanghai Jiao Tong \\ University, Shanghai 200240, China \\ ${ }^{5}$ Central Laser Facility, Science and Technology Facilities Council (STFC) \\ Rutherford Appleton Laboratory (RAL), Didcot, OX11 0QX, UK \\ ${ }^{6}$ Department of Physics, Renmin University of China, Beijing 100872, China \\ 7 Songshan Lake Materials Laboratory, Dongguan, Guangdong 523808, China \\ ${ }^{8}$ Key Laboratory for Laser Plasmas (Ministry of Education) and School of Physics \\ and Astronomy, Shanghai Jiao Tong University, Shanghai 200240, China
}

E-mail: weiminwang1@126.com, ytli@iphy.ac.cn

\begin{abstract}
We study neutron production in interactions of an intense laser pulse with solid, near-critical density and foam targets, by two-dimensional particle-in-cell simulations. We find that compared with solid and near-critical density targets, the neutron production from foam targets is more efficient because ion acceleration and ionion collisions are significantly enhanced. This is caused due to formation of ambipolar electrostatic fields among the multi-lamellas in the foam. The energy conversion efficiency from the laser pulse to the ions inside the foam target is up to $11 \%, 12$ fold higher than the one achieved with a solid target for the same laser parameters. We also find that a foam target with thinner lamellas and larger pores between the lamellas is more favorable for neutron production due to higher laser energy absorption and longer distance for ion acceleration. The number of the neutrons can reach $10^{7}$ from a foam target with a thickness of only $20 \mu \mathrm{m}$ driven by a $10^{20} \mathrm{~W} / \mathrm{cm}^{2}$ laser pulse.
\end{abstract}

Keywords: neutron sources, ion acceleration, foam targets, laser-plasma interactions

\section{Introduction}

Neutron sources have been widely applied in material testing for fusion power plants [1], high temporal and spatial resolution radiography [2-4], neutron therapy [5] and neutron resonance spectroscopy [6]. Interactions of ultra-intense laser pulses with plasma can produce compact neutron sources with short duration in sub-ps or ps range [7] and 
high-brightness [8,9]. Therefore, theses novel neutron sources have attracted broad interest and been investigated both theoretically [10-12] and experimentally [13-15]. Photonuclear $(\gamma, \mathrm{n})$ [16-18] and ion-acceleration-induced nuclear reactions [6,9] are two ways to produce neutrons using intense, short laser pulses. In laser induced photonuclear reactions, hot electrons generate $\gamma$-rays through bremsstrahlung radiations in a high $\mathrm{Z}$ material converter, and then neutrons are produced via photonuclear reactions in the converter. For ion-acceleration-induced reactions, the prerequisite for efficient neutron production is the generation of large numbers of energetic ion collisions [14]. Different target structures including single solid bulk [14,19], "pitcher-catcher" (P-C) [9, 20-22], cluster [23] targets have been investigated.

For a solid target irradiated by an intense laser pulse, the longitudinal ponderomotive force of the pulse induces an electrostatic field that accelerates the ions from the front surface [24]. Simultaneously, the ions at the rear surface of the target are also accelerated by the target normal sheath acceleration (TNSA mechanism) [25, 26]. However, only the forward-directed ions originating from the front surface contribute to the nuclear reactions through their collisions with the background cold nuclei inside the target $[11,27]$. Compared with the TNSA at the rear surface, the ions accelerated at the front surface has lower energy and efficiency. Furthermore, there is almost no further acceleration of these ions inside the cold target bulk [28] due to the large cold electron return currents $[29,30]$.

To increase neutron yields, a pitcher-catcher (P-C) double target has been adopted and demonstrated $[8,9]$. In this scheme, the first target is used to generate energetic ions and the ions enter and collide with the second target, which can efficiently produce neutrons via ion-ion collisions. It was reported in Ref. [9] that $10^{11}$ neutrons were achieved through D-D reactions when a pulse of $80 \mathrm{~J}$ and $10^{20}-10^{21} \mathrm{~W} / \mathrm{cm}^{2}$ was used [9]. In recent years, progress on improvement and control of ion beam qualities [31-35] could further enable efficient neutron production. However the size of the neutron source in a P-C scheme is at the level of millimeter [36], much larger than the equivalent source in a single target [14], provided the same laser spot size is taken. This makes the neutron source less bright. Besides, ion acceleration from the first target is significantly suppressed by proton acceleration at the target rear due to contaminants, which is difficult to avoid [20,21].

Cluster targets have also been used for neutron production [23]. When a laser pulse interacts with a cluster target, the absorbing efficiency of laser energy can approach 90\% [37], and ions can be accelerated inside the whole target volume. For clusters with radius smaller than the quivering amplitude of a free electron $\xi=\frac{a_{0} \lambda_{0}}{2 \pi}$, where $a_{0}$ is the normalized laser electric field amplitude and $\lambda_{0}$ is the wavelength, most of the extracted electrons do not come back to the cluster [38]. The main acceleration mechanism is Coulomb explosion of the ion clouds, and the maximum ion energy is typically hundreds keV/nucleon [37]. For larger-sized cluster targets, the energy of the ions accelerated by stochastic electron heating can be several $\mathrm{MeV}$ [38].

Recently much attention has been paid to improving ion acceleration with a near- 
critical density (NCD) target [39-44]. A laser pulse can penetrate deeper into such a target due to relativistically induced transparency and strong self-focusing can also occur [39]. Furthermore, the lower target density enhances the inhibition of fast electron propagation, so that more laser energy can be transferred to ions via these electrons [29, 43]. Protons close to $100 \mathrm{MeV}$ has been demonstrated in a thin foil expanding to near-critical density [34] and about $200 \mathrm{MeV}$ protons can be generated from a $2 n_{c}$ target with a laser intensity of $3 \times 10^{21} \mathrm{~W} / \mathrm{cm}^{2}$ [44]. High laser energy coupled to ions indicates an NCD target can be a candidate to enhance neutron production.

In this paper, we study effects of the internal structures of targets on neutron production. We perform two-dimensional (2D) particle-in-cell (PIC) simulations with the KLAPS code [45] and a Monte Carlo (MC) code to look at the neutron production with three types of targets, solid, NCD and foam targets. The ion acceleration and the angular, energy, temporal and spatial distributions of the neutrons produced in the targets are compared. The simulation results show that the efficient ion acceleration inside the whole bulk of a foam target can significantly enhance neutron production. The energy conversion efficiency from the laser pulse to ions accelerated inside the foam volume can reach 11.4\%, 12 times higher than the one achieved with a solid target $(0.9 \%)$ for equivalent laser parameters. The total number of neutrons as well as the number density of forward neutrons (in the laser direction) are increased by 2 orders of magnitude. The neutron number reaches $10^{7}$ in a foam target with a thickness of $20 \mu \mathrm{m}$, when a $3 \times 10^{20} \mathrm{~W} / \mathrm{cm}^{2}$, $44 \mathrm{fs}$ laser pulse is used. Larger micro-pores and thinner lamellas of the foam target are more favorable for neutron production due to more efficient ion-ion collisions.

\section{Simulation setup}

\subsection{Particle-in-Cell simulations}

We use the 2D version of the KLAPS code to study ion acceleration in pure deuterium targets with different internal structures. In our simulations, a laser pulse propagates in the $+x$ direction with a wavelength of $1 \mu \mathrm{m}$ and $p$-polarization along the $y$ direction. The pulse is normally incident on a target located from $x=22 \mu \mathrm{m}$. The laser focal spot radius (electric field waist radius) $R_{0}$ is $9 \mu \mathrm{m}$ and the pulse duration $\tau_{0}$ is $44 \mathrm{fs}$ in full width at half maximum (FWHM). The laser electric field is a normal Gaussian pulse, which is written by

$$
\begin{aligned}
\mathbf{E}\left(r, x^{\prime}\right)= & \hat{r} a_{0} \frac{R_{0}}{R\left(x^{\prime}\right)} \exp \left(\frac{-r^{2}}{R^{2}\left(x^{\prime}\right)}\right) \\
& \exp \left[-\mathrm{i} k x^{\prime}-\mathrm{i} k \frac{r^{2}}{2 x^{\prime}\left[1+\left(\frac{\pi \omega_{0}^{2}}{\lambda_{0} x^{\prime}}\right)^{2}\right]}+\mathrm{i} \phi\left(x^{\prime}\right)\right],
\end{aligned}
$$

$r$ is the radial distance from the center axis of the beam and $x^{\prime}=x-c t$. In our 2D simulations, we take the laser intensity value calculated in the $3 \mathrm{D}$ geometry. The peak intensity is $3.4 \times 10^{20} \mathrm{~W} / \mathrm{cm}^{2}$, corresponding to the amplitude $a_{0}=5$ normalized by 
$m_{e} c \omega_{0} / e$. The simulation box size is $100 \mu \mathrm{m} \times 50 \mu \mathrm{m}$ with a spatial resolution of 0.025 $\mu \mathrm{m}$ both in the longitudinal $(x)$ and transverse $(y)$ directions. 64 simulation electrons and deuterons are adopted per cell.

We use three types of targets, solid, NCD and foam ones. To allow for effects of the prepulse of an intense laser pulse, a preplasma is included in the front of the targets with a density profile linearly increasing from $0.1 n_{c}$ at $x=22 \mu \mathrm{m}$ to $1 n_{c}$ at $x=30 \mu \mathrm{m}$. In the main target area, for consistency, we keep the same average areal densities, for the three targets. The densities of the solid and NCD targets are set to be $10 n_{c}$, located within $30 \mu \mathrm{m} \leqslant x \leqslant 34 \mu \mathrm{m}$, and $2 n_{c}$, located within $30 \mu \mathrm{m} \leqslant x \leqslant 50 \mu \mathrm{m}$, respectively. To imitate the micro-structures in a foam target, which is consist of multi-micro-pores distributed irregularly, a simple model of regularly distributed lamellas (located in 30 $\mu \mathrm{m} \leqslant x \leqslant 50 \mu \mathrm{m})$ is adopted, which is shown in Fig. 1. The thickness of the lamellas $d$ is $0.1 \mu \mathrm{m}$ with a density of $10 n_{c}$, and the size of the micro-pores $L^{2}=1 \mu \mathrm{m} \times 1 \mu \mathrm{m}$. These parameters are taken according to typical foam materials $[46,47]$. The density of $10 n_{c}$ is lower than a real solid material to reduce computational expense, nevertheless it involves the similar physical process of ion acceleration and electron dynamics as a real solid density.

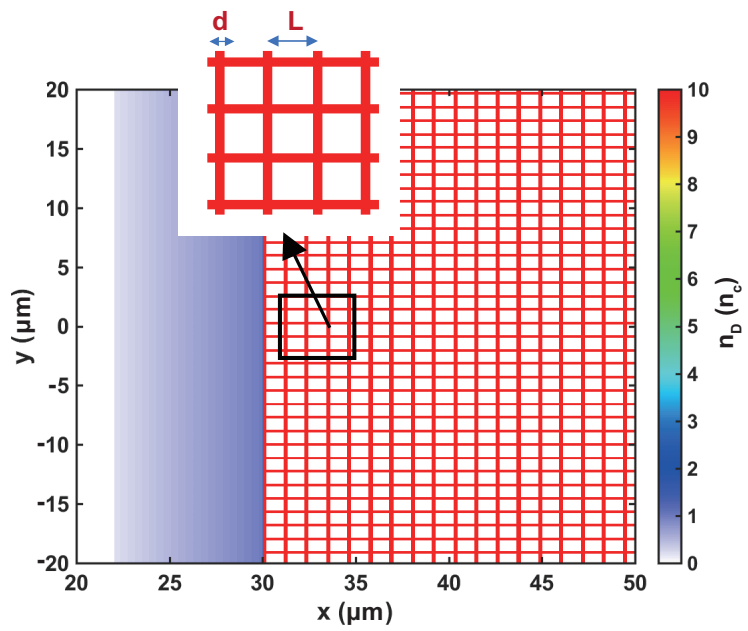

Figure 1. 2D initial density profile of the foam target. The inset illustrates inner structure parameters $d$ and $L$, representing the lamella thickness and micro-pore size, respectively.

\subsection{Monte Carlo post-processor for neutron production}

In $\mathrm{D}$ - $\mathrm{D}$ reactions $\left(\mathrm{D}+\mathrm{D} \rightarrow{ }^{3} \mathrm{He}+\mathrm{n}\right)$, the volumetric reaction rate, that is, the number of reactions per unit time and per unit volume, is given by

$$
R_{12}=n_{1} n_{2} /\left(1+\delta_{12}\right) \sigma v
$$

where $n_{1}$ and $n_{2}$ are number densities of two reacting deuteron macro-particles, $\sigma$ is the cross section, and $v=\left|\mathbf{v}_{\mathbf{1}}-\mathbf{v}_{\mathbf{2}}\right|$ is their relative velocity. $\delta_{12}$ is the Kronecker symbol 
(with $\delta_{12}=1$, if the two reacting nuclei are the same, and $\delta_{12}=0$ otherwise) [48]. The total cross section $\sigma$ is equivalent to the integration of the differential cross section $d \sigma(\theta) / d \Omega$ over the solid angle, $\sigma=\int \frac{\mathrm{d} \sigma(\theta)}{\mathrm{d} \Omega} \mathrm{d} \Omega$. In principle, the differential cross section peaks on the deuteron colliding direction and has positive correlation with colliding energy $E_{r}$ (related to relative velocity $v$ ) at any neutron emission angle $\theta_{n}$ (referred to the deuteron colliding direction). Neutron energy can be given by

$$
E_{n}=\frac{M_{D} M_{n}}{\left(M_{n}+M_{H e}\right)^{2}} E_{r}\left(\sqrt{\eta+\cos ^{2} \theta_{n}}+\cos \theta_{n}\right)^{2},
$$

where

$$
\eta=\frac{M_{n}+M_{H e}}{M_{D} M_{n}}\left(M_{H e}-M_{D}+M_{H e} \frac{Q}{E_{r}}\right),
$$

and $Q=3.266 \mathrm{MeV}$ is the reaction energy [49].

A Monte Carlo (MC) post-processor is developed to simulate the neutron production. From the PIC simulation, we randomly choose $10 \%$ of the deuterons and transfer the data of their positions, momenta and densities to the MC code. (The $10 \%$ sample is taken to reduce computational expense, while guaranteeing identical neutron energy and angular distributions.) This data transfer is performed with a time interval of $0.01 \mathrm{ps}$ in the first $1.2 \mathrm{ps}$ and $0.03 \mathrm{ps}$ after $1.2 \mathrm{ps}$. Then, the evolution of the deuteron positions, momenta and densities obtained in the PIC simulation is included in the $\mathrm{MC}$ code. In the $\mathrm{MC}$ calculation, a deuteron pair is randomly chosen from the deuterons in the same PIC cell to calculate their collision energy and reaction rate with the relative velocity $v$ of the given deuteron pair. With $v$, the differential cross sections $\mathrm{d} \sigma(\theta) / \mathrm{d} \Omega$ and total cross section $\sigma$ can be found in the tabulated data through the cubic spline interpolation. By using (2)-(4), not only the neutron numbers and energies, but also their spatial, temporal and angular distributions can be calculated, with a spatial resolution of $0.025 \mu \mathrm{m}$, the same with the PIC simulation. To estimate realistic neutron number from a 2D planar simulation, usually one can assume the laser spot size as the $z$-direction size of the produced neutron beam. Here we adopt a more reasonable method in which we take the $y$-direction size of the accelerated deuteron beam as the $z$-direction size of the neutron source.

In our case the annihilation of reacting deuterons can be neglected. In an extreme situation with the maximum cross section of 0.1 barn and a deuteron with the light speed $c$ colliding with a $10 n_{c}$ plasma, one can deduce according to (2) that the maximum reaction probability is only $7.6 \times 10^{-5}$, much less than 1 , in our whole simulation time.

\section{Results}

\subsection{Neutron production}

Figures 2 (a) and (b) show the temporal evolution of the neutron production rates $P$ (in the unit of $\mathrm{s}^{-1}$ ) and the spatial distributions of the volumetric production rates $R$ (in the unit of $\mathrm{cm}^{-3} \mathrm{~s}^{-1}$ ) along $x$-axis at $0.4 \mathrm{ps,} \mathrm{respectively,} \mathrm{where} R$ is calculated 
from (2) and $P$ is the spatial integral of $R$ over the whole targets. In the foam target, nuclear reactions occur inside the whole foam bulk [Fig. 2(b)] and $R$ is as high as $5 \times 10^{25}$ $\mathrm{cm}^{-3} \mathrm{~s}^{-1}$. The high $R$ value maintains for a long time and therefore $P$ keeps at a high level of $10^{17} \mathrm{~s}^{-1}$ from $0.1 \mathrm{ps}$ to $1.2 \mathrm{ps}$ as shown in Fig. 2(a). In both the solid and NCD targets, $R$ and $P$ have much lower levels and the nuclear reactions last shorter [Fig. 2(a)] within a smaller space [Fig. 2(b)]. Therefore, the total number of neutrons produced from the foam target is $4.4 \times 10^{5}$, much higher than the ones as $7.6 \times 10^{3}$ and $2.3 \times 10^{4}$ from the solid and NCD targets according to our PIC and MC simulations.
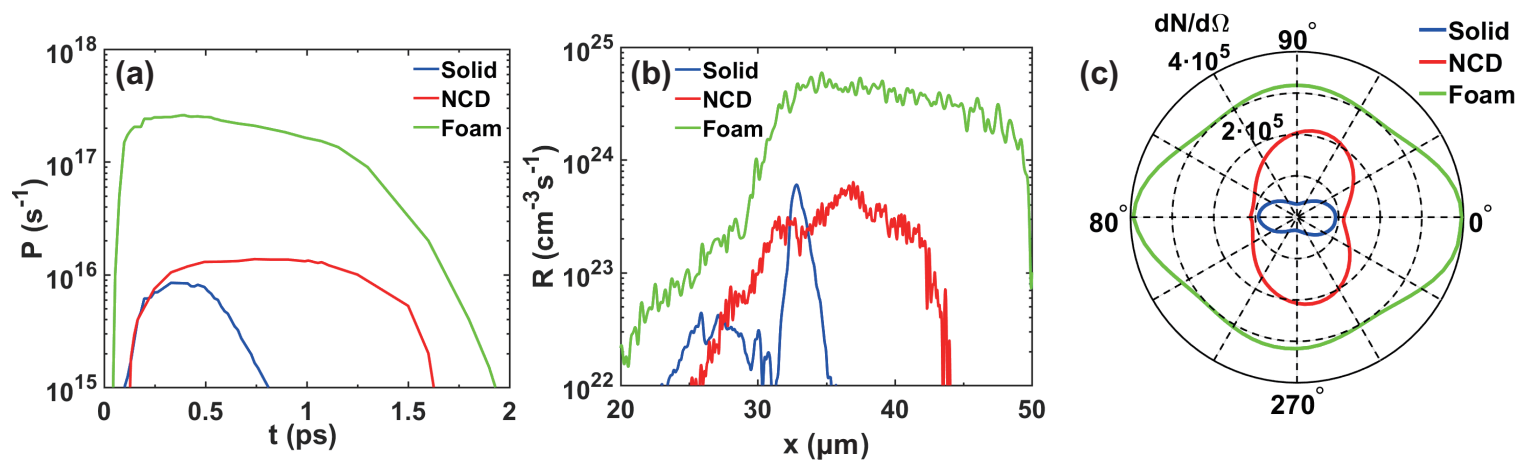

Figure 2. (a) Temporal evolution of the neutron production rates $P$; (b) longitudinal distributions of the volumetric neutron production rates $R$, which is calculated by formula (2) at 0.4 ps and averaged in $y$-direction; (c) neutron angular distributions $\left(\mathrm{d} N / \mathrm{d} \Omega\right.$ in $\left.\mathrm{MeV}^{-1}\right)$ for the solid, NCD and foam targets. The data of neutron angular distributions from the solid and NCD targets are multiplied by 10 .

Figure 2 (c) shows the neutron angular distributions. $0^{\circ}$ is the laser propagating axis (also target normal). To show it clearly, the data of the solid and NCD targets are multiplied by 10. Neutrons from the solid target is anisotropic and mainly emitted in forward and backward directions. In the NCD target, the neutron emission appears a radial distribution. For the foam target, the neutron angular distribution is quasiisotropic but stronger around $0^{\circ}$ and $180^{\circ}$. Although the neutron beam has less directivity than the solid target, the number density in the laser direction is 40 times higher.

Figure 3 shows spectra of the neutrons emitted in $0^{\circ}$ and $90^{\circ}$ from the solid, NCD and foam targets. According to Eqs.(3) and (4), when the emission angle is in $140^{\circ}$ $-180^{\circ}$, the neutron energy is around $2 \mathrm{MeV}$. As shown in Fig. 3(a) and 3(b), in $0^{\circ}$ of the solid target [blue curve in Fig. 3(a)] and $90^{\circ}$ of the NCD target [red curve in Fig. $3(\mathrm{~b})$ ], neutron spectra have peaks around $2 \mathrm{MeV}$. This indicates deuteron backward acceleration in the solid target and radial transportation in the NCD target (which is symmetrical about $x$-axis). For deuteron collisions with energy below $1 \mathrm{MeV}$, neutron emission energy is around $2.5 \mathrm{MeV}$ in $90^{\circ}-120^{\circ}$. The neutron spectrum in $90^{\circ}$ from the solid target has a peak around $2.5 \mathrm{MeV}$ [blue curve in Fig. 3(b)]. One can expect that the collisions mainly happen along $x$-axis. In the case of the foam target, peaks around 2 and $2.5 \mathrm{MeV}$ can be observed in both directions. Similar spectra in $0^{\circ}$ and 
$90^{\circ}$ indicate quasi-isotropic acceleration [green curves in Figs. 3(a) and (b)]. Also, when the deuteron spectrum and angular distribution get broader, the neutron spectrum has a slower break down at high energy, rather than a sharp cutoff. Figure 3 suggests that there is much higher deuteron energy in the foam target.
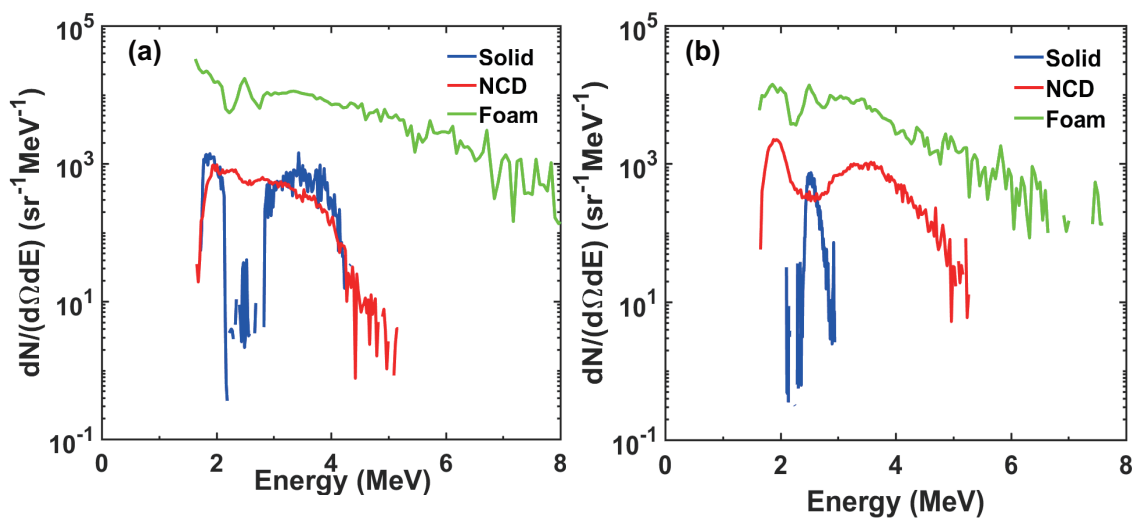

Figure 3. Spectra of the neutrons emitted at (a) $0^{\circ}$ and (b) $90^{\circ}$ from the solid, NCD and foam targets.

\subsection{Deuteron acceleration}

To further understand the neutron production presented above, we investigate the electron dynamics and deuteron acceleration in the targets. Figures 4 (a)-(c) show the laser electric fields and the electron densities $n_{e}$ in different targets at $t=0.1$ ps. Figures 4 (d)-(f) show the longitudinal electrostatic field $E_{x}$ and the deuteron densities $n_{D}$ at typical time of $t=0.07 \mathrm{ps}$ for the solid target and $t=0.13 \mathrm{ps}$ for the NCD and foam targets, respectively, when the highest $E_{x}$ appears. In the solid target, the laser propagates to the dense surface of the plasma, and is reflected there [see Fig. 4(a)]. Numerous hot electrons move forward and escape from the target rear, then a strong sheath electrostatic field is established [Fig. 4(d)]. This electrostatic field accelerates deuterons forward through the TNSA mechanism at the rear surface [25]. Due to large cold electron return currents, there is almost no electrostatic field induced inside the solid target.

In the NCD target with $2 n_{c}$, the laser pulse is able to propagate into the deep target bulk and piles up the electrons, which is shown in the solid green line in Fig. 4(b). This is caused by self-induced relativistic transparency [50] in low density plasma, which is also controlled by momentum anisotropy and average energy of electrons [51]. There are also corrugated bubbles generated due to the laser filamentation [52-54]. As the self-focusing effect occurs, the maximum pulse intensity inside the target at $t=0.1 \mathrm{ps}$ is increased to $8.5 \times 10^{19} \mathrm{~W} / \mathrm{cm}^{2}$. As a result, the NCD target absorbs much more energy from the laser pulse [52]. Whatsmore, the transport of hot electrons is more inhibited due to the low background electron density [43], and this part of energy can be transferred to deuterons. However, there is still no effective electrostatic field excited for deuteron acceleration 

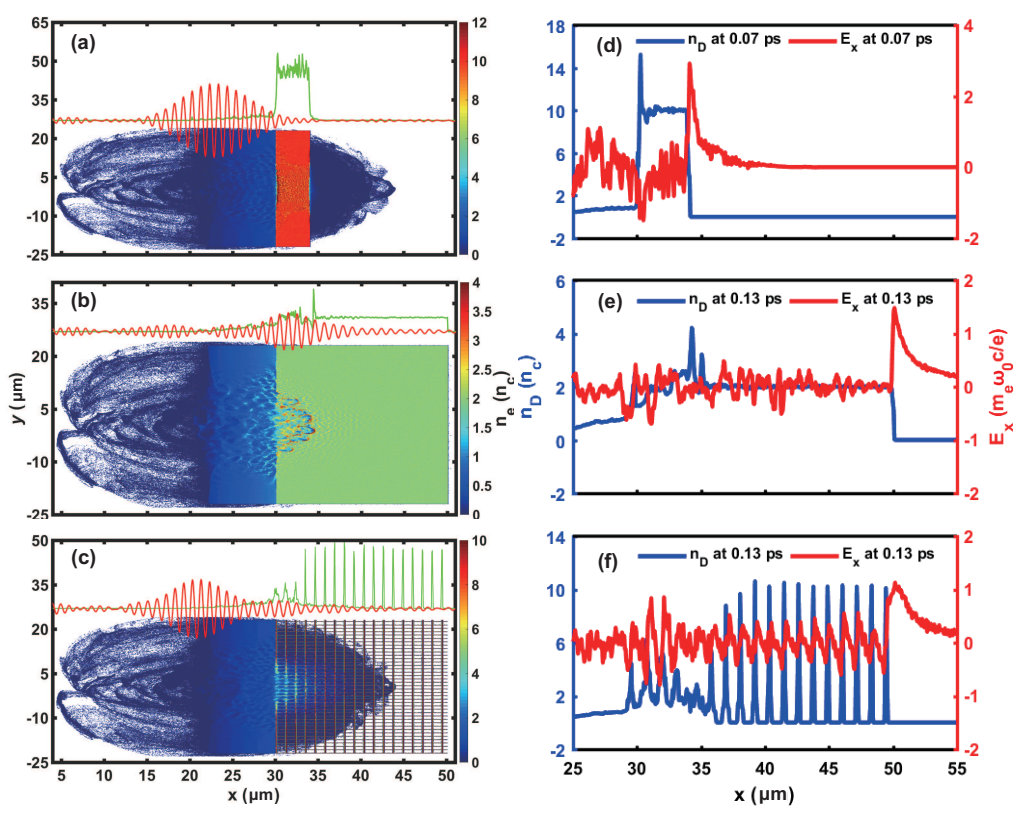

Figure 4. 2D snapshots of the electron densities $n_{e}$ at $t=0.1 \mathrm{ps}$ in the (a) solid, (b) NCD and (c) foam targets. The longitudinal distributions of the laser electric fields and electron densities at $y=0$ are shown with the red and green lines, respectively. Electrostatic fields $E_{x}$ (red lines) and deuteron densities $n_{D}$ (blues lines) in the (d) solid, (e) NCD and (f) foam targets, respectively.

inside the target, as shown in Fig. 4(e), since the density distribution is continuous and some cold electron return currents can neutralize the field. The deuteron acceleration mainly happens at the front and rear side, like the solid target.

The foam target is analogous to a multi-layered solid target. Figure 4(c) shows that the first three plasma layers are exploded and become transparent at $t=0.1$ ps, hence, the target also absorbs much laser energy. Beyond the layers reached by the laser pulse, hot electrons continue to move forward and penetrate further into the target bulk. Therefore, the background electrons in the following lamellas are heated up and expand into the micro-pores, forming ambipolar electrostatic fields among the lamellas, as shown in the red line in Fig. 4(f). Each ambipolar field will induce cascaded TNSA-like acceleration in two opposite local normal directions.

Figure 5 shows angular distribution of deuteron collision energy, where $0^{\circ}$ represents the laser incident direction. In the solid target, deuterons with relatively high energies, which contribute to D-D collision inside target, are mainly generated near the target front surface. Forward-directed ions collide with cold nuclei in background plasma and backward ions with the preplasma [shown in Fig. 5(a)]. As a result, the neutron angular distribution is anisotropy [see Fig. 2(c)]. In the NCD target, the laser pulse can penetrate deeper and bores a hole inside the target as discussed before and the radial laser pondermotive force expels electrons transversely. The deuterons are pulled by electrons radially [Fig. 5(b)]. Therefore, the neutron emission appears a radial 
distribution [Fig. 2(c)]. With the foam target, the ambipolar electrostatic fields among the lamellas [Fig. 4(f)] accelerate deuterons both forwards and backwards [Fig. 5(c) and 8(b)]. However, transverse deuteron acceleration mainly happens within a transverse size as the laser focal spot. This can explain the neutron angular distribution is quasiisotropic but stronger around $0^{\circ}$ and $180^{\circ}$, as shown in Fig. 2(c). For the solid and NCD targets, backward accelerated deuterons are less energetic than those from the foam. Since the background nuclei are under-dense in the region of preplasma ( $x$ $<30 \mu \mathrm{m})$, there are less nuclear reactions happening [see Fig. 2(b)]. In the foam target, however, due to the bulk acceleration by ambipolar electrostatic fields, energetic backward deuterons can cause higher reaction rates in the preplasma [Fig. 2(b)].
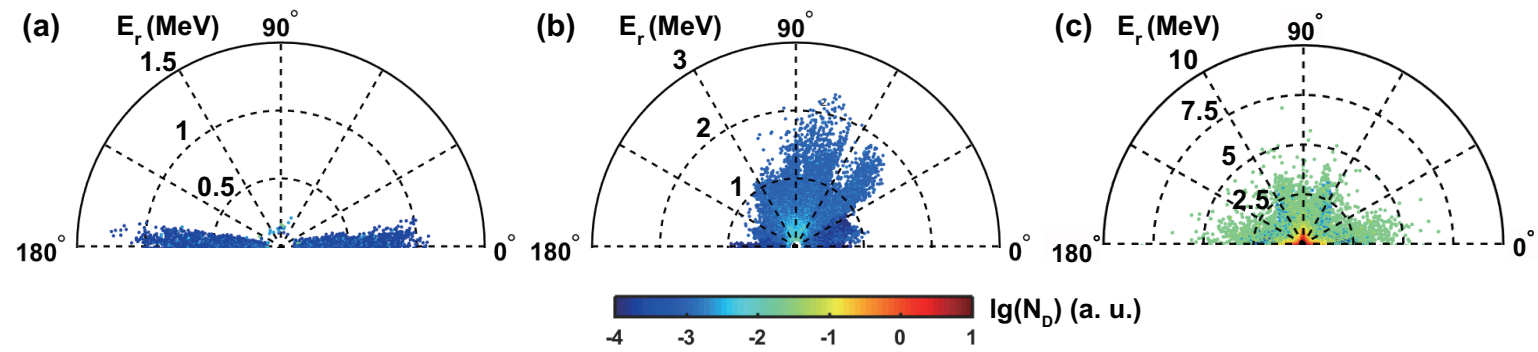

Figure 5. Angular distributions of deuteron colliding energy, where the solid, NCD and foam targets are shown in (a), (b) and (c), respectively.

The spectra of the deuterons inside the targets which contribute to the neutron production are shown in Fig. 6(a). The highest number of deuterons are generated in the foam target with cutoff energy around $4 \mathrm{MeV}$ at around 0.33 ps. The maximum collision energy is $8 \mathrm{MeV}$ as shown in Fig. 5(c), which is obtained when the colliding deuteron pairs have opposite velocities. Those collisions have higher reactivity $\sigma v$ for D-D reactions. Fig. 6(b) shows that the energy conversion efficiency from the laser pulse to the deuterons is the highest, up to $11.4 \%$, inside the foam target. The efficiencies are only $2.7 \%$ and $0.9 \%$ inside the NCD and solid targets, respectively.

\subsection{Optimization of laser and foam target parameters}

In the above sections, we have taken a foam target with $d=0.1 \mu \mathrm{m}, L=1 \mu \mathrm{m}$ and a total thickness of $20 \mu \mathrm{m}$ s shown in Fig. 1 (we call it as "Foam1"), irradiated by a $3.3 \times 10^{19} \mathrm{~W} / \mathrm{cm}^{2}$ laser pulse with a duration of $44 \mathrm{fs}$. When the duration is increased to $450 \mathrm{fs}$, the foam is heated to a relatively uniform plasma with a density around $2 n_{c}$ at the pulse end. Generally, if the pulse is too long, the bulk acceleration by ambipolar electrostatic fields could be broken down, and the neutron production inside the target would be less efficient. In the following simulations we keep the laser duration of $44 \mathrm{fs}$ and focal spot radius $9 \mu \mathrm{m}$ unchanged and change the laser energy or intensity within from $1.7 \times 10^{19} \mathrm{~W} / \mathrm{cm}^{2}$ to $3.0 \times 10^{20} \mathrm{~W} / \mathrm{cm}^{2}$.

We also take other two foam targets with the lamella and micro-pore sizes changed. We take the Foam2 with $d=1 \mu \mathrm{m}, L=1 \mu \mathrm{m}$ (thicker lamella) and Foam3 with $d=0.1$ 

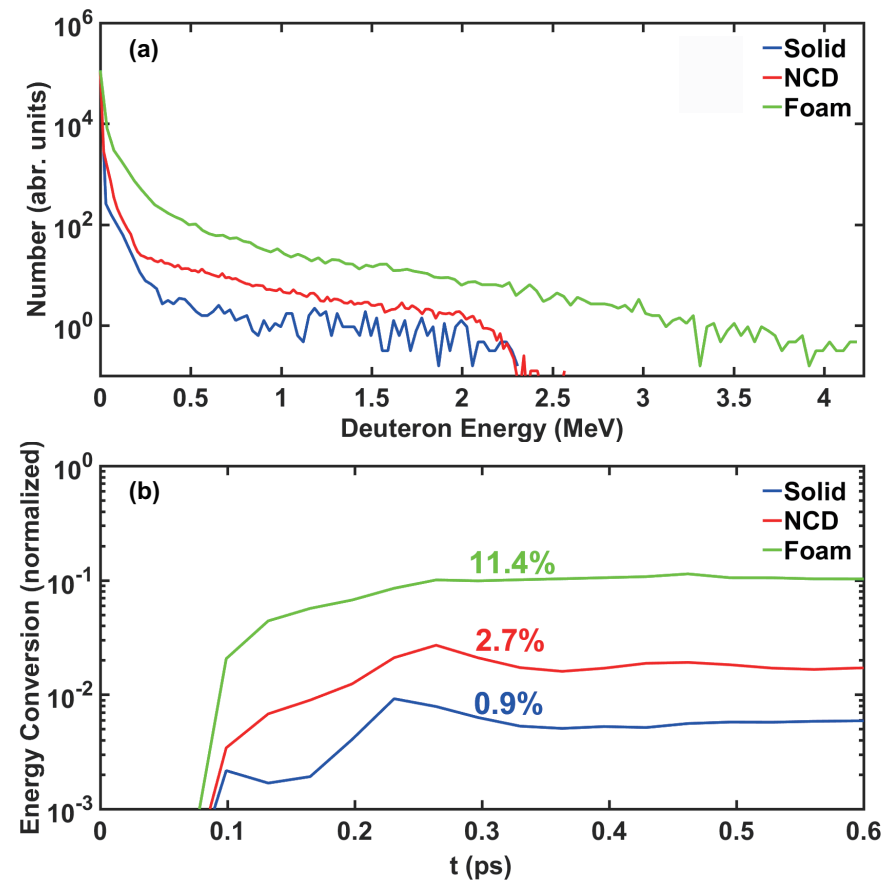

Figure 6. (a) Energy spectra of the deuterons inside the targets at $t=0.33 \mathrm{ps}$ for different targets. (b) Energy conversion efficiencies from the laser to deuterons inside the targets as a function of time.

$\mu \mathrm{m}, L=1.5 \mu \mathrm{m}$ (larger micro-pore), according to real foam materials [46, 47] and our computation resource. When $d$ or $L$ changed, the density integrals along both $x$-axis and $y$-axis are kept the same. It is shown in Fig. 7 that when larger micro-pores are adopted (Foam3), the neutron number increases with the growing laser intensity much more rapidly and the neutron production is more efficient compared with the standard Foam1. However, the neutron production becomes inefficient within a large range of the laser intensity when Foam2 with thicker lamellas is taken. These results suggest that larger pores and thinner lamella is more favorable for nuclear reactions.

Figures $8(\mathrm{a})$ and (c) show there is high density accumulation in between the lamellas in Foam2 with thicker lamellas and shorter void spaces, and Figs. 8(b) and (d) indicate deuteron colliding energy gets higher in Foam3 with thinner lamellas and larger void spaces. For Foam3, a longer acceleration distance of deuterons can be obtained for cascaded TNSA-like acceleration around each lamella. In addition, thinner lamellas in Foam3 can increase laser energy absorption, which is more favorable to deuteron acceleration. The deuteron energy enhancement causes higher neutron production according to Eq.(2).

We summarize our simulation results on different laser and target parameters in Table 1 . The anisotropy of the neutrons is estimated by the number ratio of $0^{\circ}$ and $90^{\circ}\left(N_{0^{\circ}} / N_{90^{\circ}}\right)$. When the laser intensity is increased to $3.0 \times 10^{20} \mathrm{~W} / \mathrm{cm}^{2}$, deuterons inside Foam 3 have $15 \%$ of the laser energy, and can be accelerated to $20 \mathrm{MeV}$. The total neutron number can be up to $1.1 \times 10^{7}$ within $2.5 \mathrm{ps}$. In this case, the volumetric 


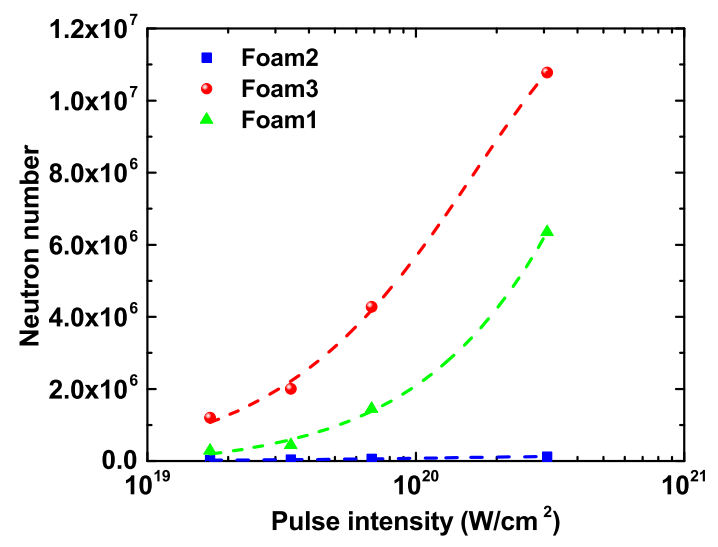

Figure 7. Neutron numbers as functions of the laser intensities in 3 kinds of foam targets
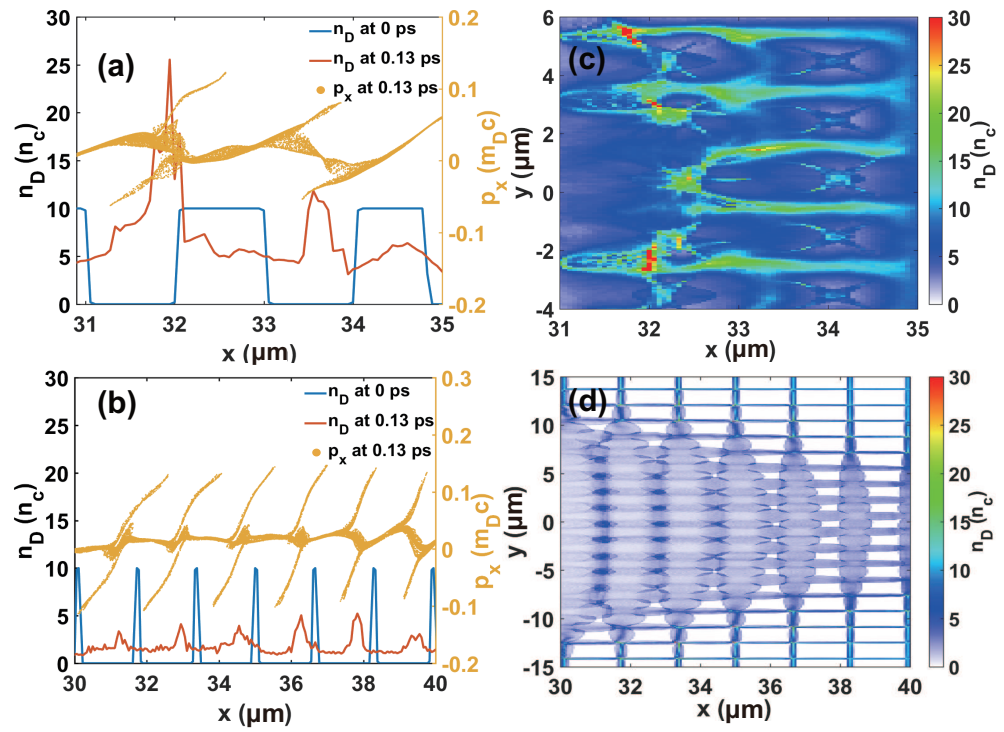

Figure 8. (a) and (b) are the longitudinal phase space (yellow points) and densities (blue and red lines for 0 and $0.13 \mathrm{ps}$ ) of deuterons in Foam2 and Foam3, where the laser intensity is taken as $3.0 \times 10^{20} \mathrm{~W} / \mathrm{cm}^{2}$. (c) and (d) are $2 \mathrm{D}$ deuteron densities of Foam 2 and Foam 3 at $t=0.13 \mathrm{ps}$, where one can see deuteron expansion, accumulation and collision inside the void spaces of foam targets.

reaction rate exceeds $3 \times 10^{28} \mathrm{~cm}^{-3} \mathrm{~s}^{-1}$, and the reaction rate reaches $8 \times 10^{18} \mathrm{~s}^{-1}$, much higher than the ones in traditional methods for neutron source production $[55,56]$. Note that we have taken foam targets with thicknesses of micrometer levels in our simulations to reduce the computing expense. One can expected that if a thick (millimeters) target is taken in a real experiment [27], the neutron number can be significantly enhanced since a smaller part of energy flux flows out from the target to the right vacuum. 
Table 1. Data related to neutron sources in different targets and laser parameters.

\begin{tabular}{lllllllll}
\hline Target $I_{L}\left(\mathrm{~W} / \mathrm{cm}^{2}\right)$ & $d(\mu \mathrm{m})$ & $L(\mu \mathrm{m})$ & $\frac{E_{D}}{E_{L}}(\%)^{a}$ & $\frac{\mathrm{d} N}{\mathrm{~d} \Omega}\left(0^{\circ}\right)\left(\mathrm{sr}^{-1}\right) \frac{\mathrm{d} N}{\mathrm{~d} \Omega}\left(90^{\circ}\right)\left(\mathrm{sr}^{-1}\right)$ & $\mathrm{d} N_{0^{\circ}} / \mathrm{d} N_{90^{\circ}}{ }^{b}$ & Neutron yield \\
\hline Solid & $3.4 \times 10^{19}$ & n.a. & n.a. & 0.9 & $9.7 \times 10^{2}$ & $4.0 \times 10^{2}$ & 2.4 & $7.6 \times 10^{3}$ \\
NCD $3.4 \times 10^{19}$ & n.a. & n.a. & 2.7 & $1.2 \times 10^{3}$ & $2.0 \times 10^{3}$ & 0.6 & $2.3 \times 10^{4}$ \\
Foam1 $3.4 \times 10^{19}$ & 0.1 & 1 & 11 & $4.0 \times 10^{4}$ & $3.3 \times 10^{4}$ & 1.2 & $4.4 \times 10^{5}$ \\
Foam1 3.0 $\times 10^{20}$ & 0.1 & 1 & 12 & $4.7 \times 10^{5}$ & $5.9 \times 10^{5}$ & 0.8 & $6.4 \times 10^{6}$ \\
Foam2 3.4 $310^{19}$ & 1 & 1 & 3.0 & $3.0 \times 10^{3}$ & $3.3 \times 10^{3}$ & 0.9 & $3.8 \times 10^{4}$ \\
Foam2 3.0 $\times 10^{20}$ & 1 & 1 & 4.4 & $1.1 \times 10^{4}$ & $9.6 \times 10^{3}$ & 1.2 & $1.2 \times 10^{5}$ \\
Foam3 3.4 $310^{19}$ & 0.1 & 1.5 & 13 & $1.9 \times 10^{5}$ & $1.5 \times 10^{5}$ & 1.3 & $2.0 \times 10^{6}$ \\
Foam3 3.0 $\times 10^{20}$ & 0.1 & 1.5 & 15 & $8.2 \times 10^{5}$ & $9.1 \times 10^{5}$ & 0.9 & $1.1 \times 10^{7}$ \\
\hline
\end{tabular}

a $\frac{E_{D}}{E_{L}}$ is the energy conversion rate from the laser pulse to deuterons inside the target.

${ }^{b} \mathrm{~d} N_{0^{\circ}} / \mathrm{d} N_{90^{\circ}}$ is the anisotropy of the neutron source represented by the ratio of neutron number per steradian at $0^{\circ}$ and $90^{\circ}$.

\section{Conclusions}

We have investigated neutron production based on the laser-driven deuteron acceleration in the solid, NCD and foam targets with the same areal density. Our 2D PIC simulations have shown that the deuteron acceleration has the highest efficiency inside the foam target than the other two, due to efficient bulk acceleration. Consequently, much stronger deuteron-deuteron collisions result in much more neutrons with higher energy produced. The neutron number is 60 times higher than the one with the solid target.

We have optimized parameters of the foam target and the laser pulse. A target with larger pores between the lamellas and thinner lamellas is more favorable to produce neutrons due to a longer distance of ion acceleration and higher efficiency of laser energy absorption. The total neutron number can be up to $10^{7}$ with only a 20 - $\mu$ m-thick foam target irradiated by a $3 \times 10^{20} \mathrm{~W} / \mathrm{cm}^{2}$ laser pulse. This work provides a method to produce high-flux short-duration neutron sources, which could find applications in fast neutron radiography and neutron scattering for material studies $[2,56,57]$.

\section{Acknowledgments}

This work is supported by the Science Challenge Project (No.TZ2016005), the National Key R\&D Program of China (Grant No.2018YFA0404801), the National Nature Science Foundation of China (Grants No.11520101003, 11827807, 11775302, 11861121001 and 11535001), the Strategic Priority Research Program of the Chinese Academy of Sciences (Grant No.XDB16010200 and XDB07030300) and the Newton foundation. 


\section{References}

[1] Perkins L J, Logan B G, Rosen M D, Perry M D, de la Rubia T D, Ghoniem N M, Ditmire T, Springer P T and Wilks S C 2000 Nuclear Fusion 401

[2] Loveman R, Bendahan J, Gozani T and Stevenson J 1995 Nuclear Instruments and Methods in Physics Research Section B: Beam Interactions with Materials and Atoms 99 765-768

[3] Anderson I S, McGreevy R L and Bilheux H Z 2009 Neutron Imaging and Applications (Springer Science+Business Media) pp 210-211

[4] Arikawa Y, Matsubara S, Abe Y, Kato Y, Kishimoto H, Yogo A, Nishimura H, Nakai M, Shiraga H, Fujioka S, Azechi H, Otake Y, Mima K and Honda Y 2017 Selected Papers from the 31st International Congress on High-Speed Imaging and Photonics 10328 103280T

[5] Gray L H and Read J 1943 Nature 15253

[6] Higginson D P, McNaney J M, Swift D C, Bartal T, Hey D S, Kodama R, Le Pape S, Mackinnon A, Mariscal D, Nakamura H, Nakanii N, Tanaka K A and Beg F N 2010 Physics of Plasmas 17 100701

[7] Macchi A 2006 Applied Physics B 82 337-340

[8] Kar S, Green A, Ahmed H, Alejo A, Robinson A P L, Cerchez M, Clarke R, Doria D, Dorkings S, Fernandez J, Mirfayzi S R, McKenna P, Naughton K, Neely D, Norreys P, Peth C, Powell H, Ruiz J A, Swain J, Willi O and Borghesi M 2016 New Journal of Physics 18053002

[9] Roth M, Jung D, Falk K, Guler N, Deppert O, Devlin M, Favalli A, Fernandez J, Gautier D, Geissel M, Haight R, Hamilton C E, Hegelich B M, Johnson R P, Merrill F, Schaumann G, Schoenberg K, Schollmeier M, Shimada T, Taddeucci T, Tybo J L, Wagner F, Wender S A, Wilde C H and Wurden G A 2013 Physical Review Letters 110044802

[10] Hu L X, Yu T P, Shao F Q, Zhu Q J, Yin Y and Ma Y Y 2015 Physics of Plasmas 22123104

[11] Toupin C, Lefebvre E and Bonnaud G 2001 Physics of Plasmas 8 1011-1021

[12] Ellison C L and Fuchs J 2010 Physics of Plasmas 17113105

[13] Torrisi L 2014 Molecules 19 17052-17065

[14] Pretzler G, Saemann A, Pukhov A, Rudolph D, Schätz T, Schramm U, Thirolf P, Habs D, Eidmann K, Tsakiris G D, Meyer-ter Vehn J and Witte K J 1998 Physical Review E 581165

[15] Abe Y, Sunahara A, Lee S, Yanagawa T, Zhang Z, Arikawa Y, Morace A, Nagai T, Ikenouchi T, Tosaki S, Kojima S, Sakata S, Satoh N, Watari T, Nishihara K, Kawashima T, Yogo A, Sakagami H, Shiraga H, Nishimura H, Mima K, Azechi H, Norimatsu T, Nakai M and Fujioka S 2017 Applied Physics Letters 111233506

[16] Ledingham K W, Spencer I I, McCanny T, Singhal R P, Santala M I, Clark E, Watts I I, Beg F N, Zepf M, Krushelnick K, Tatarakis M, Dangor A E, Norreys P A, Allott R, Neely D, Clark R J, Machacek A C, Wark J S, Cresswell A J, Sanderson D C and Magill J 2000 Physical Review Letters 84 899-902

[17] Pomerantz I, McCary E, Meadows A R, Arefiev A, Bernstein A C, Chester C, Cortez J, Donovan M E, Dyer G, Gaul E W, Hamilton D, Kuk D, Lestrade A C, Wang C, Ditmire T and Hegelich B M 2014 Physical Review Letters 113184801

[18] Jiao X J, Shaw J M, Wang T, Wang X M, Tsai H, Poth P, Pomerantz I, Labun L A, Toncian T, Downer M C and Hegelich B M 2017 Matter and Radiation at Extremes 2 296-302

[19] Disdier L, Garconnet J P, Malka G and Miquel J L 1999 Physical Review Letters 821454

[20] Maksimchuk A, Raymond A, Yu F, Petrov G M, Dollar F, Willingale L, Zulick C, Davis J and Krushelnick K 2013 Applied Physics Letters 102191117

[21] Alejo A, Krygier A G, Ahmed H, Morrison J T, Clarke R J, Fuchs J, Green A, Green J S, Jung D, Kleinschmidt A, Najmudin Z, Nakamura H, Norreys P, Notley M, Oliver M, Roth M, Vassura L, Zepf M, Borghesi M, Freeman R R and Kar S 2017 Plasma Physics and Controlled Fusion 59064004

[22] Mirfayzi S R, Alejo A, Ahmed H, Raspino D, Ansell S, Wilson L A, Armstrong C, Butler N M H, Clarke R J, Higginson A, Kelleher J, Murphy C D, Notley M, Rusby D R, Schooneveld E, 
Borghesi M, McKenna P, Rhodes N J, Neely D, Brenner C M and Kar S 2017 Applied Physics Letters 111044101

[23] Ditmire T, Zweiback J, Yanovsky V P, Cowan T E, Hays G and Wharton K B 1999 Nature 398 489

[24] Maksimchuk A, Gu S, Flippo K, Umstadter D and Bychenkov V Y 2000 Physical Review Letters 844108

[25] Wilks S C, Langdon A B, Cowan T E, Roth M, Singh M, Hatchett S, Key M H, Pennington D, MacKinnon A and Snavely R A 2001 Physics of Plasmas 8 542-549

[26] Zepf M, Clark E L, Krushelnick K, Beg F N, Escoda C, Dangor A E, Santala M I K, Tatarakis M, Watts I F, Norreys P A, Clarke R J, Davies J R, Sinclair M A, Edwards R D, Goldsack T J, Spencer I and Ledingham K W D 2001 Physics of Plasmas 8 2323-2330

[27] Willingale L, Petrov G M, Maksimchuk A, Davis J, Freeman R R, Joglekar A S, Matsuoka T, Murphy C D, Ovchinnikov V M, Thomas A G R, Van Woerkom L and Krushelnick K 2011 Physics of Plasmas 18083106

[28] Li Y T, Sheng Z M, Ma Y Y, Jin Z, Zhang J, Chen Z L, Kodama R, Matsuoka T, Tampo M, Tanaka K A, Tsutsumi T, Yabuuchi T, Du K, Zhang H Q, Zhang L and Tang Y J 2005 Physical Review E 72066404

[29] Batani D, Antonicci A, Pisani F, Hall T A, Scott D, Amiranoff F, Koenig M, Gremillet L, Baton S, Martinolli E, Rousseaux C and Nazarov W 2002 Physical Review E 65066409

[30] Bell A R, Davies J R, Guerin S and Ruhl H 1997 Plasma Physics and Controlled Fusion 39653

[31] Arefiev A, Toncian T and Fiksel G 2016 New Journal of Physics 18105011

[32] Wagner F, Deppert O, Brabetz C, Fiala P, Kleinschmidt A, Poth P, Schanz V A, Tebartz A, Zielbauer B, Roth M, Stohlker T and Bagnoud V 2016 Physical Review Letters 116205002

[33] Kar S, Ahmed H, Prasad R, Cerchez M, Brauckmann S, Aurand B, Cantono G, Hadjisolomou P, Lewis C L, Macchi A, Nersisyan G, Robinson A P, Schroer A M, Swantusch M, Zepf M, Willi O and Borghesi M 2016 Nature Communications 710792

[34] Higginson A, Gray R J, King M, Dance R J, Williamson S D R, Butler N M H, Wilson R, Capdessus R, Armstrong C, Green J S, Hawkes S J, Martin P, Wei W Q, Mirfayzi S R, Yuan X H, Kar S, Borghesi M, Clarke R J, Neely D and McKenna P 2018 Nature Communications 9724

[35] Scott G G, Carroll D C, Astbury S, Clarke R J, Hernandez-Gomez C, King M, Alejo A, Arteaga I Y, Dance R J, Higginson A, Hook S, Liao G, Liu H, Mirfayzi S R, Rusby D R, Selwood M P, Spindloe C, Tolley M K, Wagner F, Zemaityte E, Borghesi M, Kar S, Li Y, Roth M, McKenna P and Neely D 2018 Physical Review Letters 120204801

[36] Alejo A, Green A, Ahmed H, Robinson A P L, Cerchez M, Clarke R, Doria D, Dorkings S, Fernandez J, McKenna P, Mirfayzi S R, Naughton K, Neely D, Norreys P, Peth C, Powell H, Ruiz J A, Swain J, Willi O, Borghesi M and Kar S 2016 Nuclear Instruments and Methods in Physics Research Section A: Accelerators, Spectrometers, Detectors and Associated Equipment 829 176-180

[37] Esirkepov T, Bingham R, Bulanov S, Honda T, Nishihara K and Pegoraro F 2000 Laser and Particle Beams 18 503-506

[38] Breizman B N, Arefiev A V and Fomyts'kyi M V 2005 Physics of Plasmas 12056706

[39] Bulanov S S, Bychenkov V Y, Chvykov V, Kalinchenko G, Litzenberg D W, Matsuoka T, Thomas A G, Willingale L, Yanovsky V, Krushelnick K and Maksimchuk A 2010 Physics of Plasmas 17 043105

[40] Willingale L, Nilson P M, Thomas A G R, Bulanov S S, Maksimchuk A, Nazarov W, Sangster T C, Stoeckl C and Krushelnick K 2011 Physics of Plasmas 18056706

[41] Bin J H, Ma W J, Wang H Y, Streeter M J, Kreuzer C, Kiefer D, Yeung M, Cousens S, Foster P S, Dromey B, Yan X Q, Ramis R, Meyer-ter Vehn J, Zepf M and Schreiber J 2015 Physical Review Letters 115064801

[42] Willingale L, Nagel S R, Thomas A G, Bellei C, Clarke R J, Dangor A E, Heathcote R, Kaluza M C, Kamperidis C, Kneip S, Krushelnick K, Lopes N, Mangles S P, Nazarov W, Nilson P M 
and Najmudin Z 2009 Physical Review Letters 102125002

[43] Bond D J, Hares J D and Kilkenny J D 1980 Physical Review Letters 45252

[44] Nakamura T, Bulanov S V, Esirkepov T Z and Kando M 2010 Physical Review Letters 105135002

[45] Wang W M, Gibbon P, Sheng Z M and Li Y T 2015 Physical Review E 91013101

[46] Okihara S, Esirkepov T, Nagai K, Shimizu S, Sato F, Hashida M, Iida T, Nishihara K, Norimatsu T, Izawa Y and Sakabe S 2004 Physical Review E 69026401

[47] Franke D and Pahl M H 1997 Chemical Engineering and Processing: Process Intensification 36 $175-183$

[48] Atzeni S and Meyer-ter Vehn J 2004 The Physics of Inertial Fusion: BeamPlasma Interaction, Hydrodynamics, Hot Dense Matter (Oxford University Press on Demand) pp 3-4

[49] Youssef A, Kodama R, Habara H, Tanaka K A, Sentoku Y, Tampo M and Toyama Y 2005 Physics of Plasmas 12110703

[50] Palaniyappan S, Hegelich B M, Wu H C, Jung D, Gautier D C, Yin L, Albright B J, Johnson R P, Shimada T, Letzring S, Offermann D T, Ren J, Huang C, Hörlein R, Dromey B, Fernandez J C and Shah R C 2012 Nature Physics 8 763-769

[51] Stark D J, Bhattacharjee C, Arefiev A V, Toncian T, Hazeltine R D and Mahajan S M 2015 Physical Review Letters 115025002

[52] Wilks S C, Kruer W L, Tabak M and Langdon A B 1992 Physical Review Letters 691383

[53] Ma Y Y, Chang W W, Yan Y, Cao L H and Yue Z W 2001 Chinese Physics Letters 18 1628-1631

[54] Ma Y, Zhao J, Li Y, Li D, Chen L, Liu J, Dann S J D, Ma Y, Yang X, Ge Z, Sheng Z and Zhang J 2018 Proceedings of the National Academy of Sciences of the United States of America 115 6980-6985

[55] Mank G, Bauer G and Mulhauser F 2011 Reviews of Accelerator Science \& Technology 04 219-233

[56] Anderson I S, Andreani C, Carpenter J M, Festa G, Gorini G, Loong C K and Senesi R 2016 Physics Reports 654 1-58

[57] Faenov A, Matsubayashi M, Pikuz T, Fukuda Y, Kando M, Yasuda R, Iikura H, Nojima T, Sakai T, Shiozawa M, Kodama R and Kato Y 2015 High Power Laser Science and Engineering 3 e27 